\title{
National Guard Status
}

National Cancer Institute

\section{Source}

National Cancer Institute. National Guard Status. NCI Thesaurus. Code C114858.

The status of an individual who is serving in the National Guard. 